\title{
Claudicaciones en vacas de rebaños lecheros grandes y pequeños del sur de Chile ${ }^{\#}$
}

\author{
Lameness in cows from large and small dairy herds in southern Chile \\ E Flor ${ }^{\mathbf{a}}, \mathbf{N}$ Tadich $^{\mathbf{b} *}$ \\ aPrograma de Magíster en Ciencias, Mención Salud Animal, Facultad de Ciencias Veterinarias, \\ Universidad Austral de Chile, Valdivia, Chile. \\ bInstituto de Ciencias Clínicas Veterinarias, Facultad de Ciencias Veterinarias, Universidad Austral de Chile, Valdivia, Chile.
}

\begin{abstract}
SUMMARY
This study was carried out in order to determine the prevalence of lame cows, the frequency of the lesions and the degree of cow's lameness in 91 dairy farms of the $\mathrm{X}^{\text {th }}$ Region in Chile. Thirty-four large dairy herds (LDH) with 10,159 (299 \pm 57 ) cows and 57 small dairy herds (SDH) with 1,600 (28 \pm 19$)$ cows were visited once each, between August and December of 2004. During the visit the locomotion of all milking cows was observed. The degree of lameness was scored from 0 (not lame) to 4 (severe lameness). In lame cows all four feet were examined. Cow's data was recorded on individual recording sheets, being introduced as numerical variables in an Excel spread sheat, and analyzed using the statistical software Statistixs 8.0. The difference in prevalence between large and small herds and between different degrees of lameness was determined using $\chi^{2}$ Test. The prevalence of lameness was $33.12 \%$ in $\mathrm{LDH}$ and $28.7 \%$ in SDH ( $\mathrm{P}<0.02)$. From the total lame cows $50.85 \%$ and $44.05 \%$ had a lameness score $\geq 2$ in the $\mathrm{LDH}$ and SDH, respectively. The three main lesions in large farms were white line lesions (54.9\%), solear haemorrhage (52.7\%) and heel erosion (48.4\%) while in the small farms it was the white line lesions $(82.5 \%)$, heel erosion $(53.3 \%)$ and solear haemorrhage (24.6\%). Ninety-two percent of the lesions were found in the hind limbs, in both types of herds. It was concluded that there is a high prevalence of lameness in dairy farms of the $\mathrm{X}^{\text {th }} \mathrm{Region}$. The prevalence and frequency of presentation of the lesions in LDH differed from those found in SDH.
\end{abstract}

Palabras clave: claudicaciones, vacas lecheras.

Key words: lameness, dairy cattle.

\section{INTRODUCCION}

Los problemas podales son considerados como uno de los problemas más importantes en los rebaños lecheros, junto con las mastitis y problemas reproductivos (Alban y col 1996, Acuña 2002). Las cojeras afectan al bienestar animal (Whay y col 2002) y producen elevadas pérdidas económicas en la industria lechera (Alban y col 1996, Galindo y col 2000, Juárez y col 2003). Las pérdidas económicas están relacionadas con la eliminación temprana de animales, disminución del consumo voluntario de alimento, disminución de la producción de leche (Warnick y col 2001, Zimmerman 2001), alteración de los indicadores reproductivos (Alban y col 1996, Nordlund y col 2004), deterioro de la condición corporal, aumento de los costos por tratamientos y honorarios profesionales (Shearer 1998, Acuña 2002).

Las cojeras son problemas multifactoriales y difíciles de abordar, debido a que los factores de riesgo para su presentación son múltiples. Entre estos se mencionan el ambiente, manejo y construcciones (Alban y col 1996, Leonard y col 1994, Shearer 1998, Berry 2001, Bell 2004), factores

Aceptado: 08.08.2007.

\# Financiado por proyecto FONDECYT 1040176.

* Casilla 567, Valdivia, Chile; ntadich@uach.cl de origen nutricional-metabólico (Manson y Leaver 1989, Vermunt 1992, Greenough y col 1996, Ossent y Lischer 1998, Ishler y col 1999, Hoblet y col 2000, Westwood y col 2003), genéticos (Enevoldsen y col 1991, Greenough 1997, Ossent y col 1997), comportamiento (Galindo y col 2000, Ishler y col 1999, Greenough y Acuña 2002, Acuña y col 2004, Nordlund y col 2004), efecto del número ordinal del parto (Hoblet y col 2000, Hirst y col 2002, Acuña y col 2004).

Las cojeras afectan todos los aspectos del bienestar animal, ya que, dificultan el acceso al alimento y al agua, mantienen una constante incomodidad, y el dolor causado es de larga duración, impidiendo que el animal exprese su comportamiento normal (Galindo y col 2000, Green y col 2002, Whay 2002).

Diversos autores han cuantificado las pérdidas económicas producidas por las cojeras, las cuales varían de acuerdo a la patología, la disminución en producción láctea y al valor de la atención veterinaria y del litro de leche en cada país; sin embargo, todos coinciden en que estas pérdidas disminuyen la eficiencia del productor lechero (Esslemont 1990, Vermunt 1992, Alban y col 1996, Enting y col 1997, Bennett y col 1999, Bargai 2000, Greenough y Acuña 2002).

En Chile la información acerca de la prevalencia de problemas podales es escasa y obtenida de un pequeño 
número de rebaños. Vidal (1986) determinó una prevalencia de 46,6\% de alteraciones podales en tres rebaños lecheros de la provincia de Valdivia; Escobar (1993) encontró en cinco rebaños lecheros de la VIII Región una prevalencia de $11,45 \%$, mientras que Briceño (1997) señala que la frecuencia de animales con problemas podales en los meses de otoño e invierno era de $18,6 \%$ en dos rebaños lecheros de la Zona Central de Chile. Del Río (2003), en un estudio retrospectivo de las principales patologías podales en cinco rebaños lecheros de la Décima Región de Chile, encontró una prevalencia promedio de $33,4 \%$. Rodríguez y col (1998) señalan que la prevalencia de dermatitis digital en la V y VI Región de Chile en los años de 1995 y 1998 fluctuó entre un $6,1 \%$ y $10 \%$. Finalmente, Tadich y col (2005) indican que la prevalencia para rebaños de mediano tamaño de la X Región es de un 9,1\%.

En lo que respecta a los países sudamericanos, Argentina reporta que el $13 \%$ de los animales de descarte de los rebaños lecheros fue consecuencia de cojeras, cifra que se cree es similar en Uruguay (Greenough y Acuña 2002); para Brasil se describe una prevalencia de 30,3\% (Molina y col 1999) y $29,7 \%$ (Silva y col 2001).

Existen abundantes estudios en Europa y EE.UU., respecto a la prevalencia de problemas podales en vacas lecheras. La prevalencia existente en los Estados Unidos de Norteamérica entre 1993 y 1995 se mantuvo entre 8\% y $16,7 \%$ (Warnick y col 2001). En Dinamarca, Alban y col (1995) encontraron que la prevalencia de problemas podales era mayor a un 7\%. En Inglaterra, Clarkson y col (1996) describen una prevalencia de 20,6\% y Bargai (2000) indica una prevalencia de $25-60 \%$. En Holanda, Somers y col (2003) mencionan que la prevalencia de animales clínicamente cojos fue un $30 \%$.

El $90 \%$ de las lesiones que causan cojera ocurren en el miembro posterior y de estas lesiones el $68 \%$ ocurren en el dedo lateral, $12 \%$ en el dedo medial y $20 \%$ sobre la piel (Bell 2004). En el miembro anterior el $46 \%$ de las lesiones ocurre sobre el dedo medial y $32 \%$ en el dedo lateral y 22\% sobre la piel (Shearer 1998, van Amstel y col 2003, Bell 2004). Entre las lesiones más comúnmente descritas se encuentran las laminitis, úlcera plantar, enfermedad de la línea blanca, dermatitis digital, foot-rot, hiperplasia interdigital, dermatitis interdigital, doble suela, etc. (Münzenmayer 1997, Shearer 1998, Winckler y Willen 2001, Tadich y col 2005).

El objetivo de este estudio fue determinar la prevalencia de vacas cojas, grados de claudicación, distribución por rebaño y frecuencia de las principales patologías podales en 91 rebaños lecheros de la Décima Región de Chile.

\section{MATERIAL Y METODOS}

Se estudiaron 91 rebaños lecheros de la Décima Región de Chile. De estos, 34 eran rebaños grandes con una producción de leche de más de 1.000 .000 de litros/ año, dos ordeñas diarias y estabulación parcial o total de los animales durante el invierno y 57 pequeños con una producción de leche menor de 100.000 litros/año, una o dos ordeñas diarias, ordeño manual y en algunos casos con ordeña mecánica y las vacas se mantenían a pradera durante todo el año. La distribución geográfica de los rebaños se presenta en el cuadro 1.

Cuadro 1. Distribución de acuerdo a ubicación geográfica y tamaño de los 91 rebaños lecheros utilizados en el estudio.

Distribution according to geographical location and size of the 91 dairy herds used in this study.

\begin{tabular}{lccc}
\hline \multicolumn{1}{c}{ Provincias } & Grande & Pequeño & Total \\
\hline Valdivia & 16 & 28 & 44 \\
Osorno & 11 & 17 & 28 \\
Llanquihue & 7 & 12 & 19 \\
Total & 34 & 57 & 91 \\
\hline
\end{tabular}

Los rebaños lecheros fueron seleccionados por conveniencia. Su participación fue determinada considerando el tamaño del rebaño, la producción anual de leche, el interés de los propietarios por participar, la facilidades otorgadas para acceder al uso de los animales, la infraestructura existente para el examen de éstos, especialmente en los predios grandes, la presencia de caminos de acceso adecuados y la distancia a la cual se encontraban de la Universidad. La solicitud de colaboración de los propietarios se realizó a través de los médicos veterinarios asesores. Las lecherías fueron visitadas, por una sola vez durante el estudio, durante el período de agosto del 2004 y diciembre del 2004, de acuerdo a un programa previamente establecido.

El tamaño muestral fue calculado en base a una prevalencia esperada de un $10 \%$, un nivel de confianza de un $95 \%$ y una precisión de un 5\%. No se consideró para esto la correlación intraconglomerados ( $r h o$ ), debido a que ésta se desconocía. Esto dio como resultado 141 rebaños lecheros, estratificados en 57 rebaños pequeños (menos de 100 vacas), 50 rebaños medianos (entre 100 y 200 vacas) y 34 rebaños grandes (más de 300 vacas). El número de rebaños representa, aproximadamente, la distribución que estos tienen de acuerdo a su tamaño en la Décima Región. La información obtenida de los rebaños medianos ya ha sido publicada (Tadich y col 2005).

Durante la visita se inspeccionaron todas las vacas en ordeña $(\mathrm{n}=11.759)$, con la finalidad de detectar la claudicación de alguno de sus miembros. Las vacas fueron observadas a la salida de la sala de ordeña. Esta observación se efectuó con el animal caminando e inmóvil, sobre una superficie de cemento, cuando ésta estaba disponible. El grado de cojera de las vacas se determinó de acuerdo a la pauta de Sprecher y col (1997), modificada por Tadich y col (2005) de forma tal, que el grado 0 representaba a una vaca sana y el grado 1 a una vaca con una cojera leve, que en la escala de Sprecher está representada como grado 2. En los rebaños grandes, de todas aquellas vacas que pre- 
sentaban algún grado de cojera al momento de la visita se seleccionaron 20 vacas representando proporcionalmente los distintos grados de claudicación, las cuales fueron examinadas al día siguiente. En los rebaños pequeños, cuando el número de animales era menor a 20 , se observaron todas las vacas con problemas podales. Solamente seis rebaños pequeños tuvieron más de 20 vacas cojas, en ese caso solo se seleccionaron 20 animales. La decisión de examinar 20 vacas se debió a que éste era el número de animales físicamente posible de examinar al día, sin interferir con el manejo habitual del rebaño. El examen clínico y diagnóstico del tipo de lesión se realizó mediante el examen clínico individual de cada dedo (lateral y medial) de los miembros afectados (anteriores y posteriores), con la vaca de pie en un brete metálico. Las lesiones se registraron en una ficha individual, en la que se incluían los datos del propietario, fecha de la visita, número del rebaño, identificación de la vaca, grado de claudicación, miembros y dedos afectados, tipo y ubicación de la lesión, para posteriormente clasificar las distintas patologías.

Los datos fueron introducidos en una planilla Microsoft $\circledast$ Excel 2002 para realizar los análisis estadísticos de los resultados, previa verificación de todas las variables.

\section{ANALISIS ESTADISTICO}

La prevalencia se determinó dividiendo el número de vacas cojas al momento de la visita por el número total de vacas en ordeña observadas y el resultado multiplicando por 100. La prevalencia de vacas con cojera $\geq 2$ se obtuvo dividiendo el número de vacas con cojera $\geq 2$ al momento de la visita por el número total de vacas en ordeña observadas y el resultado multiplicando por 100. La frecuencia de las principales lesiones podales se obtuvo dividiendo el número de vacas con lesión por el número total de vacas cojas examinadas, multiplicando posteriormente por 100. Las diferencias entre las prevalencias, así como las diferencias entre los distintos grados de cojeras entre rebaños grandes y pequeños se analizaron mediante la prueba de Chi cuadrado $\left(\mathrm{X}^{2}\right)$.

\section{RESULTADOS}

El número total de vacas en lactancia de los rebaños grandes fue de 10.159, con un promedio de $299(57 \pm \mathrm{DE})$ animales por rebaño. Las razas que los constituían fueron predominantemente Holstein Friesian y Frisón Negro $(\mathrm{n}=31)$ y Frisón Rojo $(\mathrm{n}=3)$.

En el caso de los rebaños pequeños, estos tuvieron un total de 1.600 vacas en lactancia, el promedio de animales por rebaño fue de 28 animales (19 $\pm \mathrm{DE})$. Las razas predominantes fueron Frisón Negro y Holstein Friesian en 23 rebaños, Frisón Rojo en 33 rebaños y Jersey en un rebaño. Un $24,5 \%$ de estos rebaños ordeñaban una vez al día.
Por motivos tales como: la falta de luz, demora en la ordeña y condiciones climáticas adversas, de los 11.759 animales en producción sólo se observó un total de 10.699 vacas lecheras, de las cuales 9.101 pertenecían a los rebaños grandes y 1.598 a los rebaños pequeños.

La prevalencia promedio de vacas cojas en los rebaños grandes fue de un $32 \%$ ( $n=9.101 ; \min 17,2 \%$; $\max 56 \%$ ), significativamente mayor $(\mathrm{P} \leq 0,02)$ que la prevalencia promedio de vacas cojas $28,7 \%(\mathrm{n}=1.598$; $\min 0 \%$; $\max$ $70 \%)$ de los rebaños pequeños.

La prevalencia de vacas con grados 2,3 y 4 de claudicación en los rebaños grandes fue significativamente mayor $(\mathrm{P} \leq 0,0007)$ que la prevalencia encontrada en los rebaños pequeños (figura 1 ).

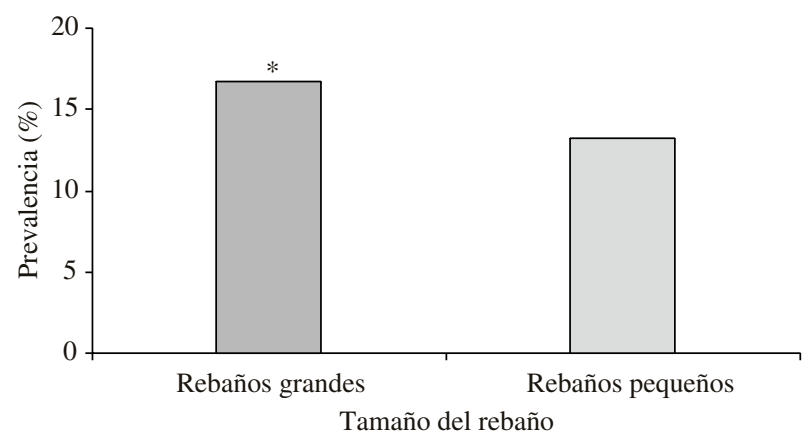

Figura 1. Prevalencia de cojeras grado $\geq 2$, en vacas de rebaños grandes $(n=9.101)$ y pequeños $(n=1.598)$ de la Décima Región, Chile. $* \mathrm{P} \leq 0,0007$.

Prevalence of lameness score $\geq 2$, in cows of large $(\mathrm{n}=9,101)$ and small $(\mathrm{n}=1,589)$ dairy herds of the $X^{\text {th }}$ Region, Chile. $* \mathrm{P} \leq 0.0007$.

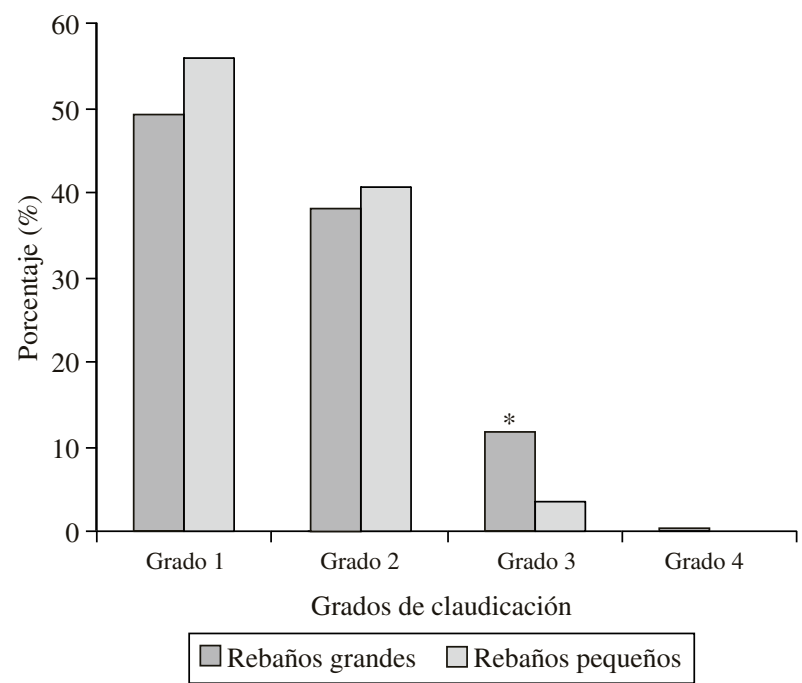

Figura 2. Distribución de los grados de claudicación (\%) en vacas cojas de 34 rebaños grandes $(n=3.014)$ y 57 rebaños pequeños $(n=458)$ de la Décima Región, Chile. * $\mathrm{P}<0,037$.

Distribution of the lameness scores $(\%)$ of cows in 34 large $(n=3,014)$ and 57 small dairy herds $(n=458)$ from the $\mathrm{X}^{\text {th }}$ Region, Chile. $* \mathrm{P}<0.037$. 
La figura 2 presenta la distribución de los grados de claudicación en las vacas cojas en los rebaños grandes y pequeños. En los rebaños grandes el porcentaje de vacas con grados de claudicación $\geq 2$ fue de un $50,85 \%$, mientras que en los rebaños pequeños fue de $44,05 \%$. Respecto al grado de claudicación 4 , solamente se encontró en los rebaños grandes $(0,17 \%)$. Sólo se observaron diferencias significativas $(P<0,037)$ entre rebaños, para los grados 3 de cojera.

Las lesiones ubicadas en los miembros posteriores fueron de un $92 \%$ para los rebaños grandes y un 91,5\% para los pequeños. El 1\% de las vacas presentaron lesiones en los miembros anteriores y el 3,5\% de animales en ambos tipos de rebaño no presentaron lesiones visibles. Sólo se encontraron animales con lesiones en los miembros anteriores en los rebaños grandes (figura 3).

El dedo lateral de los miembros posteriores fue el más afectado. Un 56,8\% de las vacas en rebaños grandes y un $61,1 \%$ en los pequeños presentaron lesiones en los dedos laterales. Un 5,6\% de las vacas en rebaños grandes y un $4 \%$ de las vacas en rebaños pequeños tuvieron lesiones en los dedos mediales. Un $23,74 \%$ de las vacas en los rebaños grandes y un $21,9 \%$ de las vacas en los rebaños pequeños presentaron lesiones en ambos dedos. Un 13,46\% y un $13 \%$ de las vacas en los rebaños grandes y pequeños, respectivamente, no presentaron lesión en ninguno de sus dedos (figura 4).

Del total de animales en los rebaños grandes, con lesiones en los miembros anteriores, el dedo medial fue el más afectado con un 38,9\%. Los dedos laterales fueron los menos afectados con un 12,5\% y el resto presentó lesiones en ambos dedos.

Las patologías más frecuentemente diagnosticadas en las vacas examinadas en los rebaños grandes fueron:

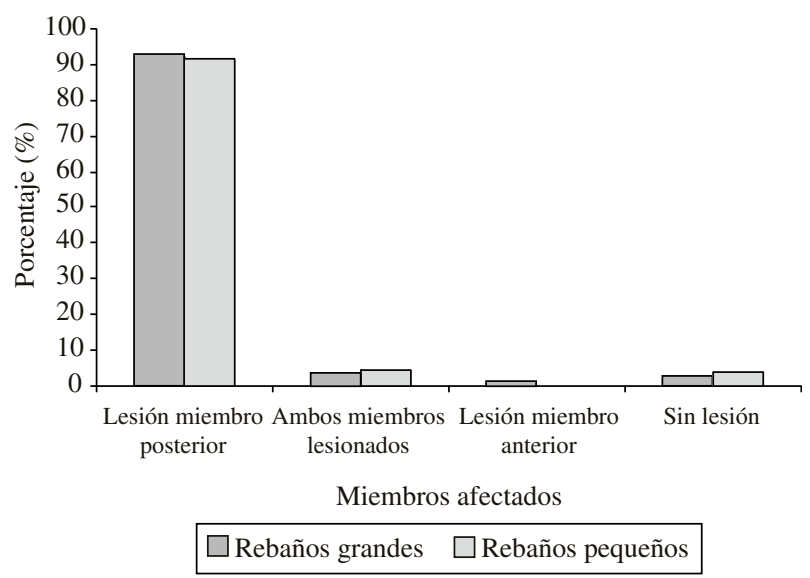

Figura 3. Distribución de las lesiones (\%), de acuerdo al miembro afectado en las vacas examinadas, en 34 rebaños grandes $(n=676)$ y 57 pequeños $(n=422)$ de la Décima Región, Chile.

Distribution of the lesions (\%), according to the affected limb in the cows examined, in 34 large $(n=676)$ and 57 small dairy herds $(n=422)$ from the $X^{\text {th }}$ Region, Chile. enfermedad de la línea blanca $(54,9 \%)$, hemorragia plan$\operatorname{tar}(52,7 \%)$, erosión de talones $(48,4 \%)$, úlcera plantar $(30,6 \%)$, doble suela $(21,4 \%)$, dermatitis digital $(7,7 \%)$ y otras $(11,4 \%)$. En tanto que en los rebaños pequeños la frecuencia de las lesiones podales más diagnosticadas fueron: enfermedad de la línea blanca $(82,5 \%)$, erosión de talones $(53,3 \%)$, hemorragia plantar $(24,6 \%)$, doble suela $(13,7 \%)$, úlcera plantar $(7,8 \%)$, dermatitis digital $(6,6 \%)$ y otras $(21,8 \%)$. En el ítem otras, se agruparon patologías con una baja prevalencia como dermatitis interdigital, abscesos de rodete coronario y de la tercera falange (figura 5).

\section{DISCUSION}

Si bien en Chile existen estudios acerca de la prevalencia de presentación de cojeras en rebaños lecheros, en todos ellos se utilizó un menor número de animales y de rebaños (Vidal 1986, Escobar 1993, Briceño 1997, Del Río 2003). Una de las restricciones de este estudio está dada por el hecho de que los rebaños no fueron seleccionados al azar, por lo que los resultados obtenidos sólo representan la situación de las cojeras en los predios lecheros grandes y pequeños estudiados. Debido a que al comenzar el estudio no se conocía el valor de rho o correlación intraconglomerados, el tamaño de la muestra se calculó sin considerar este factor, por lo que se perdió algo de poder, el cual fue de alguna manera contrarrestado por el número de rebaños utilizados al considerar una prevalencia de un $10 \%$, siendo que la prevalencia final fue de un $32,1 \%$ y un $28,7 \%$.

Debido al diseño del estudio, en el cual no se conocía la prevalencia inicial de cojeras en los predios seleccionados, el modelo de regresión logística utilizado (stepwise logistic

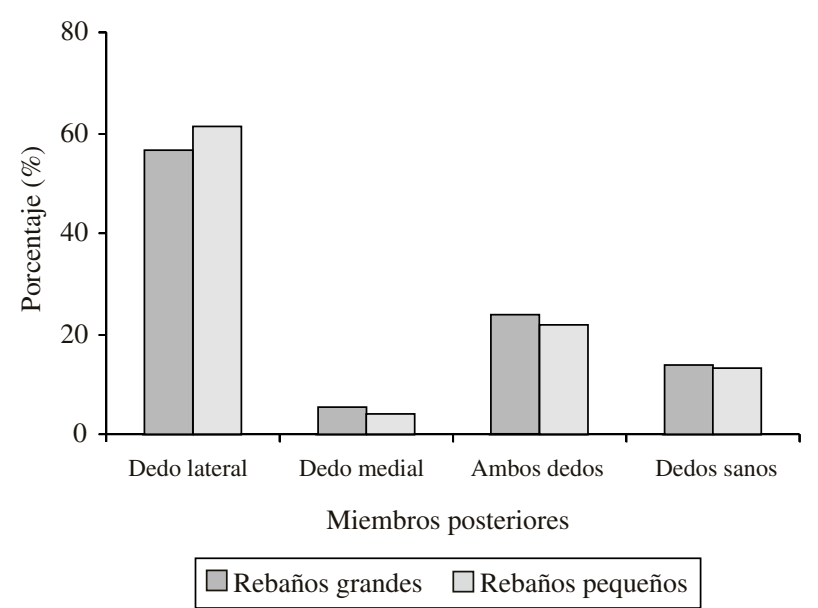

Figura 4. Distribución de las lesiones (\%), de acuerdo al dedo afectado en los miembros posteriores de las vacas examinadas, en 34 rebaños grandes $(n=676)$ y 57 pequeños $(n=422)$ de la Décima Región, Chile.

Distribution of the lesions (\%), according to the affected foot in the rear limbs of the cows examined, in 34 large $(n=676)$ and 57 small dairy herds $(n=422)$ from the $X^{\text {th }}$ Region, Chile. 


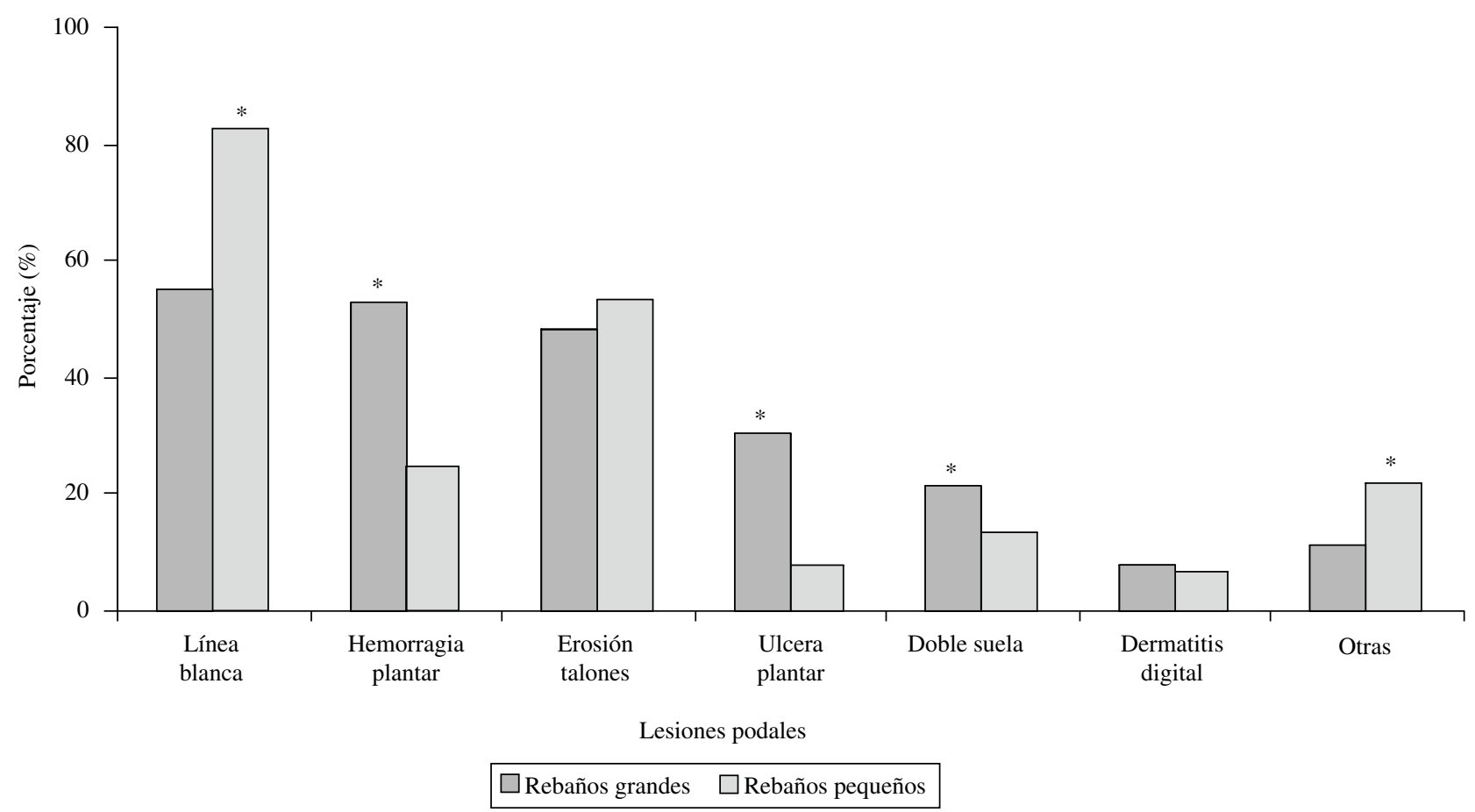

Figura 5. Frecuencia de presentación de lesiones podales en las vacas examinadas, en 34 rebaños grandes $(n=676)$ y en rebaños pequeños $(\mathrm{n}=422)$ de la Décima Región, Chile. * $(\mathrm{P} \leq 0,05)$.

Frequency of presentation of foot lesions in the cows examined, in 34 large $(n=676)$ and 57 small dairy herds $(n=422)$, from the $\mathrm{X}^{\text {th }}$ Region, Chile. * $(\mathrm{P} \leq 0.05)$.

regression) para determinar los factores asociados con altas $(\geq 15 \%)$ o bajas prevalencias $(\leq 10 \%)$ indicó que factores como el tamaño del rebaño, la suplementación con sales minerales y la limpieza diaria del patio de espera de la sala de ordeña estaban asociados a altas prevalencias. Esto podría deberse a la pérdidad de un importante número de rebaños con prevalencias entre un $10 \%$ y $15 \%$, disminuyendo de esta forma el tamaño de la muestra (Flor, 2006).

La prevalencia total encontrada en el presente estudio es mayor a las descritas por Escobar (1993), Briceño (1997) y Tadich y col (2005). Escobar (1993) en una investigación efectuada en cinco rebaños lecheros de la zona central de Chile encontró una prevalencia de 11,45\% y Briceño (1997) en un estudio de las principales patologías podales en dos rebaños lecheros de la zona central de Chile encontró una prevalencia de un 18,6\%. Ambos estudios sólo consideraron a aquellos animales que presentaban lesiones podales graves (grados $>2$ ), por lo que que la prevalencia real de animales con claudicaciones pudo haber sido subestimada. Sin embargo, los resultados de estos autores son similares a la prevalencia de cojeras grados 2,3 y 4 , encontradas en este estudio (figura 1). Tadich y col (2005) señalan una prevalencia total promedio de $9,1 \%$ en rebaños de tamaño medio; si bien dicho estudio tuvo un diseño similar a la presente investigación, existe la posibilidad de que los observadores hayan subestimado la prevalencia de los animales con grados 1 y 2 de cojera. Por otra parte, también debería considerarse la posibilidad de que en el presente estudio los grados 1 estén sobreestimados.

La prevalencia total encontrada en este estudio es similar al 33,38\% encontrado por Del Río (2003), quien realizó un estudio retrospectivo de las patologías registradas entre los años 1996-2000 en cinco rebaños lecheros de la Décima Región de Chile.

Cuando se consideran todos los grados de claudicación los resultados de este estudio son similares a los de Molina y col (1999), quienes en 10 rebaños lecheros de Belo Horizonte, Brasil, encontraron una prevalencia de $30,28 \%$, mientras que Silva y col (2001), en el Municipio de Orizona Go (Brasil) y Cruz y col (2001) en Río Grande do Sul (Brasil), registraron prevalencias entre $29,67 \%$ y $50,2 \%$, respectivamente.

La mayor parte de los estudios extranjeros presentan las prevalencias de las cojeras con un grado de claudicación $\geq 2$; aunque existen diferencias tanto en el manejo de los rebaños, número de animales en ordeña y ubicación geográfica, la mayoría de ellos describen prevalencias superiores a las obtenidas en el presente estudio para vacas con claudicaciones $\geq 2$. Es así como Clarkson y col (1996), en 37 rebaños lecheros del Reino Unido, señalan una prevalencia de 20,6\%. Sin embargo, Bargai (2000) en diversos estudios realizados en el Reino Unido encontró prevalencias entre un 25 y un $60 \%$. Esta variación puede deberse a los diversos sistemas de recolección de datos, 
las diferentes áreas de estudio y la estación del año donde fueron realizados los estudios. Somers y col (2003) en Holanda mencionan una prevalencia de animales clínicamente cojos del 30\%; Whay y col (2002), en 53 rebaños lecheros del Reino Unido, en época invernal, encontraron una prevalencia de animales clínicamente cojos de 22,1\%; Cook (2003), en 30 rebaños lecheros de Wisconsin, encontró en la estación invernal una prevalencia de animales clínicamente cojos de un 23,9\%.

En relación a la ubicación de las lesiones, los resultados de este estudio concuerdan con los trabajos de investigación realizados en Chile y en el extranjero. Shearer (1998) y Murray y col (1996) indican que el $90 \%$ de las lesiones que causan cojera ocurren en el miembro posterior. Entre los estudios de mayor importancia realizados en Chile tenemos a: Escobar (1993), Briceño (1997), Del Río (2003) y Tadich y col (2005), quienes encontraron que los miembros posteriores son los más afectados con un $83,2 \%, 90,8 \%, 86,6 \%, 82 \%$, respectivamente. Sin embargo, Vidal (1986) encontró un porcentaje menor $(62,4 \%)$ de afección de los miembros posteriores. Con respecto a los porcentajes de miembros anteriores afectados, los resultados del presente estudio difieren de los encontrados tanto en Chile como internacionalmente, lo que puede estar influido por el número de animales que fue posible examinar en los campos grandes, por lo que es posible que se haya subestimado el número de animales con lesiones en los miembros anteriores.

Referente a los dedos afectados, estos resultados concuerdan con los obtenidos en diversas investigaciones; así, Escobar (1993), Briceño (1997) y Tadich y col (2005) observaron que el $56,3 \%, 58,7 \%$ y $52,8 \%$ de las lesiones se ubicaban en los dedos laterales, del miembro posterior. En el caso de los miembros anteriores, el medial fue el más afectado, con un 9,6\%. Shearer (1998) y Murray y col (1996) señalan que para el miembro anterior el $46 \%$ de lesiones se presentan en el dedo medial, $32 \%$ en el dedo lateral y $22 \%$ sobre la piel.

$\mathrm{Si}$ bien es cierto que la cojera en el bovino se define como un problema multifactorial (Alban y col 1996, Enevoldsen y col 1991, Zimmerman 2001, Acuña 2002), existen patologías podales que se asocian a trastornos metabólicos, entre ellas, la enfermedad de línea blanca, hemorragias plantares, úlceras plantares y la doble suela. De estas lesiones, la enfermedad de la línea blanca se presentó con mayor frecuencia en rebaños grandes y pequeños con un $54,9 \%$ y un $82,5 \%$, respectivamente. Este tipo de lesión se ha relacionado con la laminitis subclínica que es de mayor presentación en rebaños tecnificados, como lo son los rebaños grandes (Clarkson y col 1996; Stone 2004). La elevada presentación de esta enfermedad en los rebaños pequeños podría atribuirse a otros factores de riesgo como son: el daño mecánico por caminar sobre superficies abrasivas o caminos en mal estado, falta de recorte funcional de las pezuñas en el ganado lechero y con ello un crecimiento excesivo de la uña (Toussaint
1985, Greenough y col 1996, Shearer y van Amstel 2000, van der Tol y col 2004).

Escobar (1993) y Tadich y col (2005) encontraron una frecuencia de la enfermedad de la línea blanca de $10,59 \%$ y $15,9 \%$, respectivamente, las cuales son menores al porcentaje de este estudio. Esto podría deberse que en los estudios mencionados anteriormente sólo se diagnosticó la ELB, cuando era el principal problema causante de la cojera, mientras que en este estudio se diagnosticaron todas las lesiones presentes en los dedos independientemente de su gravedad. Los resultados del presente estudio están más cercanos a lo informado por Eddy y Scott (1980), Baggott (1982) y Russell y Rowlands (1982), quienes señalan que en el Reino Unido la ELB es la lesión de mayor frecuencia con un 35\%, 20\% y $18 \%$ respectivamente; mientras que en Nueva Zelanda, Tranter y col (1991) señalan que la incidencia de la ELB fue del $39 \%$, siendo la lesión de mayor frecuencia en vacas que presentaban problemas podales.

La segunda lesión de mayor importancia en los rebaños grandes fue la hemorragia plantar con $52,9 \%$, mientras que en los rebaños pequeños fue de un $24,6 \%$. Esta diferencia entre los distintos tipos de rebaño podría deberse a que la hemorragia plantar se relaciona con desbalances nutricionales producidos por exceso de concentrado en la ración, y es por eso que se observa con mayor frecuencia en rebaños de alta producción lechera. Por lo general, son consecuencia de una laminitis subclínica que ha ocurrido tiempo atrás y no a una indicación del estado del animal en el momento del examen (Vermunt 1992, Hoblet y col 2000). También pueden, excepcionalmente, ser consecuencia de traumatismos o excesivo desgaste de la superficie solear cuando se encuentran en superficies duras (Tranter y Morris 1991). Del Río (2003) y Tadich y col (2005) mencionan que la distribución de hemorragias plantares es de baja frecuencia, con un $12,1 \%$ y $4,8 \%$ respectivamente, lo cual no concuerda con los resultados obtenidos en este estudio; probablemente esos estudios privilegiaron las lesiones más graves y las hemorragias plantares fueron subestimadas.

Tranter y Morris (1991) describen que en Nueva Zelanda la hemorragia plantar es la lesión con mayor presentación en la época invernal con un $42 \%$, indicando que se presenta con mayor frecuencia en los miembros posteriores. Manske y col (2002) encontraron que en Suecia un 30\% de los animales presentaban hemorragia plantar, estando la lesión presente en todos los rebaños examinados.

La erosión de los talones fue la tercera lesión de mayor importancia en los rebaños grandes, con un $48,4 \%$ y la segunda lesión de importancia en rebaños pequeños con un 53,3\%. No se encontró una diferencia significativa de esta patología entre los rebaños grandes y pequeños. Se cree que la época del año la falta de higiene, la humedad, el contacto con los purines y la estabulación son los factores predisponentes para la presentación de esta patología (Enevoldsen y col 1991, Bergsten y Pettersson 1992), creando 
un ambiente en donde el Fusobacterium necrophorum y el Bacteroides nodosus proliferan (Bergsten 2001).

En Chile, Escobar (1993) y Briceño (1997) encontraron la presencia de esta patología, pero en porcentajes menores a los de este estudio con un 6,84\% y un $28,97 \%$, respectivamente. Molina y col (1999) indican que la erosión de los talones afectó a un 48,5\% de las vacas lecheras mantenidas en confinamiento en Belo Horizonte, Brasil. Smilie y col (1999), al examinar las pezuñas de 203 vacas en 13 rebaños en Ohio, encontró que la erosión de los talones estaba presente en el $74 \%$ de los animales que se examinaron, de los cuales el 13,3\% se clasificaron como severos. Hultgren y Bergsten (2001) informaron que el $59 \%$ de 82 vacas de lecherías examinadas tenía erosiones severas de talón, mientras que Manske y col (2002) demostraron que la erosión de talones fue la patología más frecuente en rebaños lecheros de Suecia, con un $41 \%$. Baird (2004) indica que la presencia de erosión de talón es menor en las vaquillas antes de parir, pero que aumenta significativamente a inicios de lactancia, confirmando que se relaciona con los factores ambientales a que se someten los animales en lactancia. En general, los resultados de este estudio coinciden con los citados anteriormente.

La cuarta lesión de mayor importancia en los rebaños grandes fue la úlcera plantar con 30,6\%; sin embargo, para los rebaños pequeños esta lesión ocupó el sexto lugar en importancia con un $7,8 \%$. La amplia diferencia de presentación entre los rebaños en estudio se podría deber a que los rebaños grandes son más susceptibles a presentar laminitis, ya que en la mayoría de ellos los animales eran de alta producción y esto generalmente va acompañado de medidas de manejo más intensivas, como estabulación, mayor frecuencia de ordeño y consumo de raciones con mayor cantidad de concentrado. La laminitis produce la formación de tejido córneo de mala calidad, descenso de la tercera falange y crecimiento acelerado del tejido córneo y como resultado de este proceso se da la presencia de las úlceras plantares (Shearer y van Amstel 2000, Acuña y col 2004).

Otros estudios realizados en Chile están más de acuerdo con la frecuencia de presentación encontrada para los rebaños pequeños. Es así que Escobar (1993), Briceño (1997), Del Río (2003) y Tadich y col (2005) encontraron que la úlcera plantar tenía una baja frecuencia de presentación, esto es: $4 \%, 9,6 \%, 6,1 \%$ y $4,5 \%$, respectivamente. Sin embargo, los resultados de este estudio, para los rebaños grandes son similares a las investigaciones realizadas internacionalmente. Clarkson y col (1993) encontraron que la úlcera plantar fue la lesión de mayor frecuencia de presentación con un $28 \%$, valores similares fueron encontrados por Murray y col (1996). Kossaibati y Esslemont (2000), en 50 rebaños lecheros del Reino Unido mantenidos a estabulación libre y pastoreo, encontraron que la úlcera plantar tuvo una presentación de un 20\%; Petrie y Ferguson (2002) en un estudio en Alemania, señalan que la patología de mayor presentación fue la úlcera plantar con $28,3 \%$, indicando que la incidencia de esta lesión pudo haber sido mayor si se hubiesen incluido los meses de invierno.

La doble suela fue la quinta lesión de mayor frecuencia en los rebaños grandes con $21,4 \%$ y un $13,7 \%$ en los rebaños pequeños, siendo significativamente diferentes entre ambos grupos; esto podría estar relacionado a episodios de laminitis o a cambios bruscos en la ración que recibe el animal en los rebaños de mayor producción (Greenough y col 1996; Greenough 2002; Acuña y col 2004). Del Río (2003) y Tadich y col (2005) encontraron que la frecuencia de la doble suela fue de un 5,8\% y un $10 \%$ respectivamente, lo cual concuerda con lo encontrado en los rebaños pequeños, dado que en ellos los cambios de alimentación son menores, comparado con lo que sucede en los rebaños grandes. Bergsten (2000), Petrie y Ferguson (2002) y Bell (2004) obtuvieron resultados con rangos entre $14,5 \%$ y $31 \%$, mientras que Murray y col (1996) y Manske y col (2002) obtuvieron valores de un 2\% y 3\%, respectivamente. Estas diferencias podrían reflejar un manejo nutricional inadecuado, como también la falta de recorte preventivo que se debe realizar constantemente en los rebaños lecheros (Toussaint 1985).

La dermatitis digital fue la sexta lesión de mayor importancia en rebaños grandes y la séptima en rebaños pequeños; esta enfermedad infecciosa está muy relacionada con la higiene de los rebaños. En los rebaños grandes su frecuencia fue de un 7,7\%, muy similar a la encontrada en los rebaños pequeños $6,6 \%$. En Chile se han realizado otras investigaciones acerca de la dermatitis digital, todas con resultados similares. La investigación más reciente y realizada en la Décima Región fue la de Tadich y col (2005), en rebaños de tamaño mediano cuyo resultado fue $5,7 \%$. Rodríguez y col (1998) en un estudio realizado en la V y VI Región de Chile señalan una prevalencia del $10 \%$ y $6,1 \%$, respectivamente. Internacionalmente son varias las investigaciones realizadas: en el Reino Unido, Murray y col (1996), Kossaibati y Esslemont (2000), Hedges y col (2001) encontraron una prevalencia que oscila entre el 8 y el 25\%; en Holanda, Barkema y col (1994), encontraron una prevalencia del 21\%; en América del Norte, Warnick y col (2001) y Cook y col (2004) encontraron una prevalencia del $32 \%$ y $57 \%$, respectivamente. Se puede observar la amplia diferencia entre los valores encontrados en Chile con respecto a los valores de Europa y América del Norte, lo que podría deberse a los distintos sistemas de producción. En Chile y en especial en la Décima Región el pastoreo es la base de la producción lechera, mientras que en América del Norte y Europa el sistema productivo se basa en la estabulación fija o estabulación libre, en donde los animales están en mayor contacto con la humedad y suciedad, lo cual es un factor predisponente para la presentación de esta patología.

Con respecto a los grados de claudicación, el grado 1 representa una cojera muy leve, mientras que los grados $\geq 2$ representan una cojera moderada a grave, siendo uno de los problemas más importantes en relación al bienestar animal (Whay y col 2002), produciendo elevadas pérdi- 
das económicas en la industria lechera (Alban y col 1996, Galindo y col 2000, Juárez y col 2003). En este estudio se encontró que en los rebaños grandes la mitad de las vacas cojas presentaban un grado de claudicación $\geq 2$, mientras que en los rebaños pequeños este porcentaje fue menor $(44,1 \%)$. Tadich y col (2005) encontraron que un $46,6 \%$ de las vacas examinadas tenían grados de claudicación $\geq 2$. El hecho de que los rebaños pequeños presentaran menor porcentaje de vacas con grado 3 y 4 podría deberse a que estas vacas, por sus menores niveles de producción, son menos exigentes en el manejo nutricional, como también al hecho de que en rebaños pequeños las vacas reciban una mayor observación por parte de los propietarios. Es importante señalar el bajo porcentaje de vacas con claudicaciones grado 4 , en ambos tipos de rebaño. Esto indica que existe preocupación por parte de los ganaderos para que sus animales no lleguen a estas condiciones extremas.

En base a los resultados se concluye que la prevalencia de cojeras en rebaños grandes y pequeños fue alta, siendo significativamente mayor en los rebaños grandes. Estas prevalencias fueron superiores a las reportadas en la mayoría de estudios realizados anteriormente en el país.

Se observaron diferencias significativas entre los rebaños grandes y pequeños, en relación a la presentación de las principales patologías podales diagnosticadas. Las patologías podales observadas fueron similares a las descritas en la literatura, pero la frecuencia de presentación de éstas fue superior a las descritas en otros estudios nacionales e internacionales. Las patologías de origen infeccioso tuvieron una baja prevalencia.

Los miembros más afectados fueron los posteriores, $\mathrm{y}$ de éstos, los dedos laterales presentaron mayor número de lesiones.

\section{RESUMEN}

Se determinó la prevalencia de vacas cojas, la frecuencia de las patologías podales y el grado de claudicación en 91 rebaños lecheros en la Décima Región de Chile. Treinta y cuatro rebaños lecheros grandes (RLG), con un tamaño de rebaño promedio de $299 \pm 57$ vacas y 57 rebaños pequeños (RLP), con un tamaño de rebaño promedio de $28 \pm 19$ vacas, fueron visitados por una sola vez, entre agosto y diciembre del 2004. En la visita se observó la locomoción de las vacas en ordeña, clasificándose de 0 (sana) a 4 (muy coja). En las vacas cojas se examinaron los cuatro miembros. Los datos fueron registrados en fichas individuales e incorporados como variables numéricas a una plantilla Excel y analizados mediante el programa Statistixs 8.0. Las diferencias entre las prevalencias de los RLG y RLP y entre los distintos grados de claudicación, se determinaron mediante la prueba de $\chi^{2}$. Se estudiaron 10.159 y 1.600 vacas en RLG y RLP, respectivamente. La prevalencia total de vacas cojas en RLG fue de $33,12 \%$ y $28,7 \%$ en los RLP (P<0.02). En los RLG un $50,85 \%$ de las vacas cojas presentaron una claudicación $\geq 2$, y un $44,05 \%$ en los RLP. Las principales patologías en los RLG fueron: enfermedad de la línea blanca $(54,9 \%)$, hemorragia plantar $(52,7 \%)$ y erosión de talones $(48,4 \%)$; en los RLP: enfermedad de la línea blanca (82,5\%), erosión de talones (53,3\%) y hemorragia plantar (24,6\%). Un $92 \%$ de las lesiones causantes de cojeras afectaron los miembros posteriores, en ambos tipo de rebaños. Existe una alta prevalencia de vacas cojas en los rebaños lecheros de la Décima Región. La prevalencia y frecuencia de presentación de las lesiones fueron diferentes en los RLG, comparados con los RLP.

\section{AGRADECIMIENTOS}

Al Dr. José Borkert por su ayuda en la recolección de antecedentes durante la ejecución del proyecto.

\section{REFERENCIAS}

Acuña R. 2002. Estudio de rengueras en rodeos lecheros. Una guía para el veterinario asesor. Resúmenes del $X$ Congreso Latinoamericano de Buiatría XXX, Jornadas Uruguayas de Buiatría. Uruguay, Pp 44-53.

Acuña R, J Junqueira, K Nordlund, J Ramos. 2004. Fisiología y profilaxis. En: Cojeras del bovino. Fisiopatología y profilaxis. $1^{\mathrm{a}} \mathrm{ed}$. Interamericana, Buenos Aires, Argentina.

Alban L, JF Agger, LG Lawson. 1996. Lameness in tied Danish dairy cattle: the possible influence of housing systems, management, milk yield, and prior incidents of lameness. Prev Vet Med 29, 135-149.

Alban L, LG Lawson, JF Agger. 1995. Foul in the foot (interdigital necrobacillosis) in Danish dairy cows, frequency and possible risk factors. Prev Vet Med 24, 73-82.

Baird L. 2004. Development of hoof pathologies in dairy cattle with and without access to pasture. Thesis Master of Science (Animal Welfare Program), The University of British Columbia, Canada.

Baggott D. 1982. Hoof lameness in dairy cattle. In Practice 4, 133-141.

Bargai U. 2000. Lameness in a dairy herd: An Epidemiologic Model. Cont Educ Pract 22, 58-67.

Barkema HW, JD Westrik, K van Keulen, Y Schukken. 1994. The effects of lameness on reproductive performance, milk production and culling in Dutch dairy farms. Prev Vet Med 20, 249-259.

Bell E. 2004. Description of claw horn lesions and associated risk factors in dairy cattle in the lower Fraser Valley, British Columbia. Thesis Master of Science (Animal Welfare Program). The University of British Columbia, Canada.

Bennett R, K Chistiansen, R Clifton-Hadley. 1999. Estimating the costs associated with endemic diseases of dairy cattle. $J$ Dairy Research $66,455-459$.

Bergsten C. 2000. Laminitis in practice: causes, risk factors, treatment and prevention. Proceedings 2000 Hoof Health Conference. Duluth, USA, Pp 57-65.

Bergsten C. 2001. Laminitis: causes, risk factors, and prevention. Mid-south Ruminant Nutrition Conference. Texas, USA, Pp 57-67.

Bergsten C, B Pettersson. 1992. The cleanliness of cows tied in stalls and the health of their hooves as influenced by the use of electric trainers. Prev Vet Med 13, 229-238.

Berry S. 2001. Diseases of the digital soft tissues. The Veterinary Clinics of North America. Food Animal Practice 17, 129-142.

Briceño C. 1997. Estudio de las patologías podales más frecuentes en dos lecherías de la zona central del país durante otoño e invierno. Memoria de titulación, Escuela de Ciencias Veterinarias, Universidad de Chile, Chile.

Clarkson MJ, D Downham, W Faull, J Hughes, F Manson, J Merritt, R Murray, W Russell, J Sutherst, W Ward, M Thrusfield. 1993. An epidemiological study of dairy cattle lameness. Society for Veterinary Epidemiology and Preventive Medicine, Proceeding of a meeting held at the University of Exeter, UK.

Clarkson MJ, D Downham, W Faull, J Hughes, F Manson, J Merritt, R Murray, W Russell, J Sutherst, W Ward. 1996. Incidence and prevalence of lameness in dairy cattle. Vet Rec 138, 563-567.

Cook N. 2003. Prevalence of lameness among dairy cattle in Wisconsin as a function of housing type and stall surface. J Am Vet Med Assoc 223, 1324-1328.

Cook N, K Nordlund, G Oetzel. 2004. Environment influences on claw horn lesions associated with laminitis and subacute ruminal acidosis in dairy cows. J Dairy Sci 87, E36-E46.

Cruz C, G Driemeier, C Cerva, L Corbellini. 2001. Clinical and epidemiological aspect of bovine digital lesions in southern Brazil. Arq Bras Med Vet Zootec 53, 654-657. 
Del Río MJ. 2003. Caracterización retrospectiva de las principales patologías podales en cinco rebaños lecheros con sistemas productivos semiintensivos, de la X Región, Chile. Memoria de titulación, Facultad de Medicina Veterinaria, Universidad de Concepción, Chile.

Eddy R, C Scott. 1980. Some observations on the incidence of lameness in dairy cattle in Somerset. Vet Rec 106, 140-144.

Enevoldsen C, T Grohn, I Thysen. 1991. Heel erosion and other interdigital disorders in dairy cows: associations with season, cow characteristics, disease, and production. J Dairy Sci 74, 1299-1309.

Enting H, D Kooij, A Dijkhuizen, R Huirnr. 1997. Economic losses due to clinical lameness in dairy cattle. Livest Prod Sci 49, 259-267.

Escobar CR. 1993. Prevalencia de patologías podales claudicantes en vacas lecheras de alta producción en confinamiento permanente, en la comuna de San Carlos, VIII Región. Memoria de titulación, Facultad de Medicina Veterinaria, Universidad de Concepción Chile.

Esslemont RJ. 1990. The cost of lameness in dairy herds. Proceedings of the VI International Symposium on Diseases of the Ruminant Digit, Liverpool, UK, Pp 237-251.

Flor E. 2006. Claudicaciones en vacas de rebaños lecheros de la Décima Región, Chile: Prevalencia, lesiones y factores de riesgo. Tesis Magíster en Ciencias mención Salud Animal. Fac. de Cs. Veterinarias, Universidad Austral de Chile.

Galindo F, DM Broom, G Jackson. 2000. A note on possible link between behaviour and the occurrence of lameness in dairy cows. $J$ Appl Anim Behaviour Sci 67, 335-341.

Green L, VJ Hedges, YH Schukken. 2002. The impact of clinical lameness on the milk yield of dairy cows. J Dairy Sci 85, 2250-2256.

Greenough P. 1997. Understanding herd lameness. Western dairy Management Conference, Las Vegas, Nevada. USA, Pp 97-106.

Greenough P. 2002. Lesiones que afectan la región digital del bovino. Resúmenes del X Congreso Latinoamericano de Buiatría, XXX Jornadas Uruguayas de Buiatría, Uruguay, Pp 59-67.

Greenough P, R Acuña. 2002. Bases epizootiológicas de las claudicaciones en rodeos lecheros. Resúmenes del $X$ Congreso Latinoamericano de Buiatría, XXX Jornadas Uruguayas de Buiatría, Uruguay, Pp 54-58.

Greenough P, V Schugel, B Johnson. 1996. Manual ilustrado sobre Problemas de patas en Bovinos. En: Zimpro Corporation, Philadelphia, USA, Pp 30.

Hedges J, RW Blowey, AJ Packington, CJ O'Callaghan, LE Green. 2001. A longitudinal field trial of the effect of biotin on lameness in dairy cows. J Dairy Sci 84, 1969-1975.

Hirst W, RD Murray, WR Ward, P French. 2002. A mixed-effects time-to-event analysis of the relationship between first-lactation lameness and subsequent lameness in dairy cows in the UK. Prev Vet Med 54, 191-201.

Hoblet K, RH Smilie, L Midla. 2000. Subclinical laminitis in dairy cattle: maintaining healthy hoof horn. Food Animal Practice 22, 97-107.

Hultgren J, C Bergsten. 2001. Effect of a rubber-slatted flooring system on cleanliness and foot health in tied dairy cows. Prev Vet Med 52, 75-89.

Ishler V, D Wolfgang, D Griswold. 1999. Prevention and control of foot problems in dairy cows. Department of Dairy and Animal Science and Department of Veterinary Science, College of Agricultural Sciences and Cooperative Extension, The Pennsylvania State University, USA, Pp 1-20.

Juarez S T, P H Robinson, EJ De Peters, O Price. 2003. Impact of lameness on behavior and productivity of lactating Holstein cows. J Appl Anim Behaviour Sci 83, 1-14.

Kossaibati MA, RJ Esslemont. 2000. The incidence of lameness in 50 dairy herds in England. Proceedings of the III International Conference on Bovine Lameness. Parma, Italia, Pp 160-162.
Leonard F, J O'Connell, K O'Farrell. 1994. Effect of different housing conditions on behavior and foot lesions in Friesian heifers. Vet Rec 134, 490-494.

Manske T, J Hultgren, C Bergsten. 2002. Prevalence and interrelationships of hoof lesions and lameness in Swedish dairy cattle. Prev Vet Med 54, 247-263.

Manson F J, JD Leaver. 1989. The influence of dietary protein intake and hoof trimming on lameness in dairy cattle. Anim Prod 47, 191.

Molina L, A Carvalho, E Facury, P Ferreira, V Ferreira. 1999. Prevalência e classificação das afecções podais em vacas lactantes na bacia leiteira de Belo Horizonte. Arq Brás Méd Vet Zootec 51.

Münzenmayer W. 1997. Afecciones podales en rodeos lecheros: desafío profesional. Suplemento especial. Therios, 25-29.

Murray RD, DY Downham, MJ Clarkson, WB Faull, JW Hughes, FJ Manson, JB Merritt, WB Russell, JE Sutherst, WR Ward. 1996. Epidemiology of lameness in dairy cattle: description and analysis of foot lesions. Vet Rec 138, 586-591.

Nordlund K, N Cook, G Oetzel. 2004. Investigation strategies for laminitis problem herds. J Dairy Sci 87, (E. suppl) E27-E35.

Ossent P, P Greenough, J Vermunt. 1997. Laminitis. In: Greenough P (ed). Lameness in cattle. $3^{\text {rd }}$ ed. Saunders Company, England, Pp 277-292.

Ossent P, C Lischer. 1998. Bovine laminitis: the lesions and their pathogenesis. In Pract 20, 415-427.

Petrie L, JG Ferguson. 2002. An evaluation of lameness in a large dairy herd in the state of Sachsen-Anhalt, Germany. Proceedings of the XII International Symposium on Lameness in Ruminants. Florida, USA, Pp 343-345.

Rodríguez A, P Meléndez, D Hird, D Read. 1998. Papillomatous digital dermatitis in Chilean dairies and evaluation of a screening method. Prev Vet Med 37, 197-207.

Russell A, G Rowland.1982. Survey of lameness in British dairy cattle. Vet Rec 111, 155-160.

Shearer JK. 1998. Lameness in dairy cattle: consequences and causes. The Bovine Practitioner 32, 79-84.

Shearer JK, S van Amstel. 2000. Lameness in dairy cattle. Dairy Western Dairy Management Conference, Kentucky, USA, Pp 1-12.

Silva LAF, LM Silva, AF Romani, RE Rabelo, MC Soares, T Mendes de Souza, C Da Silva. 2001. Características clínicas e epidemiológicos das enfermidades podais em vacas lactantes do Municipio de Orizona-Go. Ciencia Animal Brasileira 2, 119-126.

Smilie RH, KH Hoblet, ML Eastridge, WP Weiss, GL Schnitkey, ML Moeschberger. 1999. Subclinical laminitis in dairy cows: use of severity of hoof lesions to rank and evaluate herds. Abstract. Vet Rec 144, 17-21.

Somers J, K Frankena, E Noordhuizen Stassen, J Metz. 2003. Prevalence of claw disorders in Dutch dairy cows: exposed to several floor systems. J Dairy Sci 86, 2082-2093.

Sprecher JD, ED Hostetler, BJ Kaneene. 1997. A lameness scoring system that uses posture and gait to predict dairy cattle reproductive performance. Theriogenology 47, 1179-1187.

Stone W. 2004. Nutritional approaches to minimize subacute ruminal acidosis and laminitis in dairy cattle. J Dairy Sci 87 (E. Suppl.) E13-E26.

Tadich N, E Hettich, G van Schaik. 2005. Prevalencia de cojera en vacas de 50 rebaños lecheros del sur de Chile. Arch Med Vet 37, 29-36.

Toussaint Raven E. 1985. Cattle footcare and claw trimming. Farming Press Ltd, The Netherlands.

Tranter WP, RS Morris. 1991. A case study of lameness in three dairy herds. New Zeal Vet J 39, 88-96.

Tranter WP, RS Morris, N Williamson. 1991. A longitudinal study of the hooves of non-lame cows. New Zeal Vet J 39, 53-57.

Van Amstel SR, JK Shearer, PL Palin. 2003. Case report-clinical response to treatment of pododermatitis circumscripta (ulceration of the sole) in dairy cows. The Bovine Practitioner 37, 143-150.

Van der Tol P, S van der Beek, J Metz, EN Noordhuizen-Stassen, W Back, C Braam, W Weijs. 2004. The effect of preventive trimming 
on weight bearing and force balance on the claws of dairy cattle. $J$ Dairy Sci 87, 1732-1738.

Vermunt J. 1992. Subclinical laminitis in dairy cattle. New Zeal Vet J 40, 133-138.

Vidal R. 1986. Estudio de las afecciones podales en hembras bovinas de lechería de tres rebaños de la Provincia de Valdivia. Memoria de titulación, Escuela de Medicina Veterinaria, Universidad Austral de Chile, Chile.

Warnick LD, LC Janssen, CL Guard, YT Grohn. 2001. The effect of lameness on milk production in dairy cows. J Dairy Sci 84, 1988-1997.

Westwood C, E Bramley, I Lean. 2003. Review of the relationship between nutrition and lameness in pasture-fed dairy cattle. New Zeal Vet J 51, 208-218.
Whay H. 2002. Locomotion scoring lameness detection in dairy cattle. In Practice 24, 444-449.

Whay H, D Main, L Green, J Webster. 2002. Farmer perception of lameness prevalence. Proceedings of the 12th International Symposium on Lameness in Ruminants. Orlando, Florida, USA, January 9-13, Pp 355-358.

Winckler Ch, S Willen. 2001. The reliability and repeatability of a lameness scoring system for use as an indicator of welfare in dairy cattle. J Anim Sci Suppl 30, 103-107.

Zimmerman A. 2001. Lameness in dairy cattle: are activity levels, hoof lesions and lameness correlated? Thesis Master of Science (Animal Welfare Program). The University of British Columbia, Canada. 